\title{
DETERMINACIÓN DE LA DEMANDA MUNDIAL DE PRODUCTOS AGRÍCOLAS BANANO, CACAO VERSUS LOS DE PRIMERA NECESIDAD, MAÍZ, SOJA, TRIGO Y ARROZ. DISEÑNO DE UN MODELO ECONOMÉTRICO PARA EL PERÍODO DE 1988-2015
}

\author{
DETERMINATION OF THE GLOBAL DEMAND FOR AGRICULTURAL PRODUCTS \\ BANANAS, CACAO VERSUS THE FIRST NECESSITY, CORN, SOYA, WHEAT, \\ AND RICE. DESIGN OF AN ECONOMETRIC MODEL \\ FOR THE PERIOD 1988-2015
}

\section{FREDDY CAMACHO VILLAGOMEZ1', CHRISTIAN BERMÚDEZ GALLEGOS² ${ }^{2}$ YANINA BAJAÑA VILLAGOMEZ ${ }^{3}$}

\footnotetext{
Universidad Católica de Santiago de Guayaquil. freddy.camacho@cu.ucsg.edu.ec

2 Universidad Católica de Santiago de Guayaquil. christian.bermudez@cu.ucsg.edu.ec

3 Universidad Católica de Santiago de Guayaquil. yanina.bajana@cu.ucsg.edu.ec
}

Se explica la demanda de los productos agrícolas suntuarios versus los de primera necesidad a nivel mundial. Se analiza como los de primera necesidad ante recesiones mundiales, se siguen consumiendo mientras que los suntuarios se dejan de consumir. Se expone también su variación en el consumo de los hogares. El estudio es longitudinal, cuantitativo y correlacional. El método utilizado fue la recopilación de datos. Las fuentes oficiales consultadas fueron, la Organización de las Naciones Unidas para la Agricultura y la Alimentación (de sus siglas en inglés FAO), Common format for Transient Data Exchange for power systems (COMTRADE) y Fondo Monetario Internacional (FMI), cada una de ellas para la construcción de las variables en estudio, en series de tiempo desde 1988 hasta 2015, y consecuentemente el análisis de los resultados obtenidos. La metodología se enmarca dentro de las cuatro etapas de la investigación, que más adelante se presentarán de forma específica. En la primera etapa se determina el precio ponderado a nivel mundial de los productos analizados; en la segunda se calcula la Yield (rendimiento proporcional de los beneficios de una actividad económica), variable instrumental que corrige por el lado de la oferta sus variaciones; en la tercera se obtiene el ingreso per cápita y el costo de vida ponderado por el nivel de importaciones de cada país; $y$ finalmente, se plantea la regresión que permitirá llegar a las conclusiones. Por lo tanto, se concluyó que los coeficientes del precio, el Producto Interno Bruto (PIB) per cápita y el costo de vida son variables que explican de manera significativa la demanda de los productos como la soja, banano y cacao.

PALABRAS CLAVE: demanda, precios, Producto Interno Bruto (PIB) per cápita, costo de vida.

\section{ABSTRACT}

It explains the demand for sumptuary versus primary agricultural products worldwide. It is analyzed as the staples of world recessions, are still consumed while the sumptuous are stopped consuming. It also shows its variation in household consumption. The study has a longitudinal, quantitative and correlational horizon. The method used was data collection. The official sources consulted were the Food and Agriculture Organization of the United Nations (FAO), the COMTRADE and the International Monetary Fund (IMF), each for the construction of variables under study, in time series since 1988 until 2015, and consequently the analysis of the results obtained. The methodology is framed within four stages of the research, which will be presented later in a more specific way. In the first stage the global weighted price of the products analyzed is determined; in the second Yield is calculated, instrumental variable that corrects by the side of the offer its variations; in the third one, we obtain the per capita income and cost of living weighted by the level of imports of each country, and finally, the regression that will allow reaching the conclusions. Therefore, it was concluded that the coefficients of price, GDP per capita and cost of living are variables that significantly explain the demand for products such as soybean, bananas and cocoa.

KEYWORDS: demand, prices, per capita GDP, cost of living. 
INTRODUCCIÓN

La investigación que genera el presente artículo, analizó el comportamiento de la demanda de los productos de primera necesidad arroz, maíz, soja y trigo, con respecto a los suntuarios banano y cacao considerados, estos últimos, entre los principales productos de exportación del Ecuador.

En la actualidad, la demanda de los productos alimenticios se ha vuelto de gran interés mundial, sobre todo en los países en desarrollo preocupados por la seguridad alimentaria. La misma, ha sufrido cambios, debido a diferentes motivos como son las recesiones económicas a nivel mundial. Esta situación, ha conllevado a un decrecimiento de los ingresos de los hogares. Así mismo, la oferta ha sido alterada por los cambios climáticos, evento que afecta particularmente a los pequeños agricultores que son los más vulnerables a este tipo de factores.

Los hogares a nivel mundial sufren las consecuencias del aumento en los costos de vida a causa de la inflación. Por tal motivo, el valor real del dinero produce cambios y las personas tienden a comprar menos con el mismo salario.

Debido a estos factores se vuelve importante determinar la demanda de los productos agrícolas en términos mundiales. Países como Ecuador producen bienes suntuarios y sería relevante para los hacedores de políticas económicas de los países en desarrollo, ver el comportamiento de la demanda, así como para tomar medidas de protección de los agricultores.

PLANTEAMIENTO DEL PROBLEMA

Tras la culminación de la segunda guerra mundial, los países con altos ingresos han experimentado un crecimiento económico, que ha ido de la mano con importantes transformaciones en los hogares (aumento del Producto Interno Bruto (PIB) - per cápita y disminución del número de personas en los hogares). Los países de la Organización para la Cooperación y el Desarrollo Económicos (OCDE) representan el 75\% de los mercados de destino de las exportaciones de países latinoamericanos (Von Hesse, 1994).

El período de la posguerra ha estado marcado por, un incremento sostenible del porcentaje del ingreso per cápita, una disminución del número de personas en el tamaño de los hogares y por la incorporación de la mujer en el campo laboral. Estas tres características vienen de la mano con el desarrollo económico y a su vez con los hábitos de consumo alimenticios que han influido en la demanda de productos agrícolas; es decir que ante mayores ingresos de los hogares, sus miembros tienden a consumir alimentos frescos y saludables, preocupándose de su salud y nutrición (Von Hesse, 1994).

A medida que el ingreso de los individuos aumenta, la proporción del gasto va perdiendo importancia relativa. Esta relación, es conocida comúnmente como la Ley de Engel (Von Hesse, 1994). Además, el aumento del consumo de alimentos en las naciones industrializadas se debe a dos razones: (a) mayor importancia del gasto en alimentos fuera de casa, y (b) el deseo de consumir alimentos de alta calidad, pero que sean de fácil y rápida preparación. Estos cambios en los hábitos de consumo en los países industrializados han provocado que las exportaciones de los países latinoamericanos sean básicamente, de productos frescos.

Sin embargo, el balance de los últimos años es negativo para el sector de la agricultura, sobre todo en los países en desarrollo. Esta situación se encuentra relacionada con los precios de intercambio y con la debilidad de los mercados con respecto a las exportaciones (Di Girolamo, 1992).

Durante los últimos años, numerosos países han estado afectados por motivos económicos, cambios climáticos, entre otros. Esta situación ha ocasionado un sin número de recesiones mundiales que llevan consigo una disminución de los ingresos y consumo de las familias de diferentes productos, lo cual afecta al Producto Interno Bruto. En este sentido, se hace imperioso realizar un análisis, que comprenda el entorno mundial en el cual Ecuador se encuentra inmerso, y cómo se ve afectado por la globalización (Camacho, 2014).

De acuerdo a los últimos datos estimados por Banco Mundial (2014), se proyecta que el crecimiento poblacional de 2025 a 2030 aumentará en un $0,8 \%$. La calidad de un producto viene dada por los precios. Esto tiene dos componentes: (a) interno y (b) externo; que influyen significativamente en la imagen de los productos ante el consumidor, es decir hace referencia al país donde fue elaborado (Albert y Gómez, 1996). En numerosas ocasiones los consumidores perciben que lo producido fuera del país, es de mejor calidad que lo nacional. Esta percepción es recurrente en Ecuador.

A lo largo de su historia, el Ecuador, ha mantenido como promedio una balanza comercial negativa, dado que el nivel de importaciones es mayor al nivel de exportaciones. Como consecuencia, resulta una disminución del PIB, ante los diferentes factores externos, como 
disminución de la demanda de los productos agrícolas debido a las recesiones económicas y cambios climáticos.

Teniendo en cuenta estos antecedentes, es importante determinar la demanda de los productos agrícolas y las variables que la afectan debido a que también influye en la oferta de los mismos.

\section{PROPÓSITO DE LA INVESTIGACIÓN}

El propósito de la investigación cuantitativa que sirvió de base al presente artículo, fue comprender los cambios que se han originado en el transcurso de los últimos años en la producción y consumo de productos agrícolas, de acuerdo a las cifras del Ministerio de Agricultura, Ganadería, Acuacultura y Pesca de Ecuador. Estos, han originado variaciones en los ingresos y en el consumo de los hogares a nivel mundial de los productos de primera necesidad. Por esta razón, se hace relevante realizar un análisis concerniente al mercado agrícola ecuatoriano con respecto al mercado mundial en lo que se refiere a productos de primera necesidad.

Por lo tanto, realizar esta investigación permitió analizar el comportamiento empírico del mercado agrícola tanto a nivel mundial como local. Con ello se podrán tener alternativas y tomar decisiones para proteger, mediante políticas económicas, a los pequeños agricultores y su producción.

FORMULACIÓN DEL PROBLEMA DE INVESTIGACIÓN ¿Qué relación existe entre la demanda de los productos agrícolas banano, cacao y los de primera necesidad trigo, soja, arroz y maíz en función del precio, PIB per cápita y costo de vida?

OBJETIVO GENERAL

Determinar la demanda mundial de productos agrícolas banano, cacao versus los de primera necesidad maíz, soja, trigo y arroz. Diseño de un modelo econométrico para el período de 1988-2015.

\section{OBJETIVO ESPECÍFICOS}

- Determinar los precios, ingresos per cápita y costo de vida de cada uno de los productos, dividiendo las exportaciones en términos monetarios de dólares para las toneladas exportadas y obteniendo un precio ponderado de cada uno de los productos a nivel mundial.

- Plantear la demanda de los productos agrícolas en relación al precio, ingreso y costo de vida, por medio de herramientas estadísticas para la comparación de los productos analizados.

- Comparar los resultados obtenidos diferenciando los productos primarios y secundarios en función de los ingresos en los hogares, a través de una matriz comparativa.

\section{PREGUNTAS DE INVESTIGACIÓN}

Las preguntas específicas de investigación

- ¿El precio tiene influencia en la demanda del banano, cacao, trigo, soja, arroz y maíz?

- ¿El PIB per cápita tiene influencia en la demanda de banano, cacao, trigo, soja, arroz y maíz?

- ¿El costo de vida tiene influencia en la demanda de banano, cacao, trigo, soja, arroz y maíz?

\section{METODOLOGÍA}

En el presente artículo, se detalla el procedimiento efectuado sobre las series temporales de costo de vida, volumen de exportaciones, precio de exportación, PIB per-cápita y rendimiento de arroz, banano, cacao, maíz, soja y trigo.

DESARROLLO DEL MÉTODO ESTADÍSTICO PROPUESTO Como se ha descrito anteriormente, la investigación planteó en términos matemáticos una regresión de la forma:

$Q_{d}=\beta_{0}+\beta_{1}$ Precio $+\beta_{2}$ Rentapercapita $+\beta_{3}$ Costodevida $+\varepsilon$

Dónde:

$Q_{d} \quad=$ Cantidades demandas

$P \quad=$ Precio de los productos

Renta = Ingreso per cápita de cada país

Costo de Vida = Variación del costo de vida a nivel mundial

$\varepsilon=$ Error de la regresión

Cabe recalcar que todas las variables están medidas en términos de toneladas y han sido obtenidas a través de páginas oficiales como la COMTRADE, FAO y el FMI.

Los datos se han obtenido de tal manera, que se puede minimizar el error y se han analizado siguiendo el esquema detallado a continuación:

a. Estadística descriptiva de cada variable.

b. Regresión multivariada incluyendo todos los predictores.

c. Análisis de resultados obtenidos. 
COSTO DE VIDA

En cuanto al costo de vida, se utilizaron los datos del FMI a nivel mundial, en donde se detalla el costo de vida y la ponderación por importaciones de la misma. Se tomó como medida del costo de vida mundial, el promedio ponderado por nivel de importación de determinado cultivo (arroz, banano, cacao, maíz, soja y trigo), en donde el promedio ponderado del costo de vida se encuentra definido por:

$\overline{C V}_{t}=\frac{\sum_{i=1}^{n} C V_{i}^{t} * W_{i}^{t}}{\sum_{i=1}^{n} W_{i}^{t}}=\frac{C V_{1}^{t} * W_{1}^{t}+C V_{2}^{t} * W_{2}^{t}+\ldots+C V_{n}^{t *} W_{n}^{t}}{W_{1}^{t}+W_{2}^{t}+\ldots+W_{n}^{t}}$

En donde:

$\overline{C V}$ representa el costo de vida promedio en el período t.

$C V_{i}^{t}$ representa el costo de vida del país i en el período t.

$W_{i}^{t}$ representa el peso ponderado por importaciones del país i en el período t.

$w_{1}^{t}+w_{2}^{t}+\ldots+w_{n}^{t}$ representa la suma de los pesos ponderadores del país 1 al n (en donde la suma es uno).

VOLUMEN DE EXPORTACIONES

Esta variable se refiere al peso en toneladas de las exportaciones de los cultivos a analizar (arroz, banano, cacao, maíz, soja y trigo). Se encuentran calculados a partir de la FAO y consiste en la suma anual de peso volumétrico de los cultivos exportados a nivel mundial. Este valor no se encuentra ponderado.

PRECIO DE EXPORTACIÓN

Esta variable se refiere al precio de exportación, expresado en dólares por tonelada, de los cultivos a analizar. Es calculada a partir de la FAO (cálculo del precio) y consiste en la división del volumen de exportaciones en dólares y el peso volumétrico (en toneladas) del cultivo.

\section{PIB PER-CÁPITA}

Con respecto a la variable PIB per-cápita se utilizaron los datos del FMI a nivel mundial, en donde se detalla la producción per-cápita y la ponderación por importaciones de la misma. Se tomó como medida del PIB per-cápita mundial, al promedio ponderado por nivel de importación del determinado cultivo (arroz, banano, cacao, maíz, soja y trigo), en donde el promedio ponderado de la producción se encuentra definido por:

$\overline{P I B}_{t}=\frac{\sum_{i=1}^{n} p i b_{i}^{t} * W_{i}^{t}}{\sum_{i=1}^{n} W_{i}^{t}}=\frac{p i b_{1}^{t} * W_{1}^{t}+p i b_{2}^{t} * W_{2}^{t}+\ldots+p i b_{n}^{t} * W_{n}^{t}}{W_{1}^{t}+W_{2}^{t}+\ldots+W_{n}^{t}}$

En donde:

$\overline{P I B_{t}}$ representa la producción per-cápita promedio en el período $t$.

$p i b_{i}^{t}$ representa la producción per-cápita del país $i$ en el período $t$.

$w_{i}^{t}$ representa el peso ponderado por importaciones del país i en el período $t$.

$w_{1}^{t}+w_{2}^{t}+\ldots+w_{n}^{t}$ representa la suma de los pesos ponderadores del país 1 al $n$ (en donde la suma es uno).

RENDIMIENTO DE CULTIVO (YIELD)

En cuanto al rendimiento medido en hectogramos por hectárea, se utilizaron los datos (Rendimiento de cada producto por hectárea), que fueron calculados a partir de la fuente oficial como COMTRADE, en donde se detalla el rendimiento por hectárea de cada uno de los cultivos a investigar (arroz, banano, cacao, maíz, soja y trigo). Este rendimiento se pondera por el nivel de importaciones de dicho cultivo. El promedio ponderado de la producción se encuentra definido por:

$\overline{\operatorname{YIELD}}_{t}=\frac{\sum_{i=1}^{n} \text { yield }_{i}^{t} * W_{i}^{t}}{\sum_{i=1}^{n} W_{i}^{t}}=\frac{\text { yield }_{1}^{t} * W_{1}^{t}+\text { yield }_{2}^{t} * W_{2}^{t}+\ldots+\text { yield }_{n}^{t} * W_{n}^{t}}{W_{1}^{t}+W_{2}^{t}+\ldots+W_{n}^{t}}$

En donde:

$\overline{Y I E L D}$ representa la productividad promedio en el período $t$.

yield $_{i}$ representa la productividad del país i en el período $t$ de un determinado cultivo.

$w_{i}^{t}$ representa el peso ponderado por exportaciones del país i en el período t.

$w_{1}^{t}+w_{2}^{t}+\ldots+w_{n}^{t}$ representa la suma de los pesos ponderadores del país 1 al $n$ (en donde la suma es uno).

RESULTADOS

A continuación se presentan el modelo econométrico planteado. Es decir, las seis regresiones 
TABLA 1. COEFICIENTES DEL PRECIO DE LOS PRODUCTOS

\begin{tabular}{lrrrrrrr|} 
& COEF. & STD. ERR. & $\mathbf{Z}$ & P>|Z| & \multicolumn{2}{c}{ [95\% CONF .INTERVAL] } & R -CUADRADO \\
\hline log_precio_arroz & -853.383 & 8.479 .241 & -1.01 & 0.314 & -2.515 .284 & 8.085 .177 & $0 \%$ \\
\hline log_precio_maíz & 2.569 .617 & 7.808 .799 & 0.33 & 0.742 & -1.273 .535 & 1.787 .458 & $0 \%$ \\
log_precio_trigo & 9.458 .691 & 1.036 .262 & 0.09 & 0.927 & -1936.45 & 2.125 .624 & $0 \%$ \\
\hline log_precio_soja & 1.378 .523 & 0.581374 & 2.37 & 0.018 & 0.2390512 & 2.517 .995 & $94 \%$ \\
\hline log_precio_banano & -3.722 .274 & 1.085 .792 & -3.43 & 0.001 & -5.850 .386 & -1.594 .161 & $32 \%$ \\
\hline log_precio_cacao & -0.4938963 & 0.2434742 & -2.03 & 0.043 & -0.971097 & -0.0166955 & $79 \%$ \\
\hline
\end{tabular}

de los productos analizados con sus respectivos resultados:

Regresión del arroz

$$
\begin{gathered}
\mathrm{Q}(\text { demanda })=24.01-8.53 \text { precio } \\
+1.04 \text { costo vida }+3.66 \text { PIB } \\
\text { Regresión del maíz }
\end{gathered}
$$

$\mathrm{Q}($ demanda $)=-106.26+25.69$ precio- 1.39

costo vida-8.70 PIB

Regresión del trigo

$\mathrm{Q}($ demanda $)=-310.42+94.58$ precio -5.93

costo vida -21.70 PIB

Regresión de la soja

$\mathrm{Q}($ demanda $)=8.95+1.37$ precio +0.10 costo vida- 0.92 PIB

Regresión del banano

$\mathrm{Q}($ demanda $)=19.89-3,72$ precio +0.48

costo vida $+0,13 \mathrm{PIB}$

Regresión del cacao

$\mathrm{Q}($ demanda $)=13.45-0,49$ precio +0.17

costo vida- $0.54 \mathrm{PIB}$

Como se puede observar, se encuentran calculados los precios, ingresos per cápita y costos de vida de cada uno de los productos analizados partir de las bases obtenidas en la FAO y el FMI.

A partir de los resultados, se analizó que las regresiones de maíz, trigo y arroz no aportan al modelo debido a que presentan $\mathrm{R}$ cuadrado del $0 \%$ de explicación con respecto a la variable dependiente; además de la poca significancia de los betas, mayor a un $\alpha=0,05 \%$ establecido.

Con respecto a la soja, banano y cacao se podría concluir que sí aportan a las regresiones planteadas con una participación mayor del $\mathrm{R}$ cuadrado. Además, cada una de las variables tiene un nivel de significancia menor de $0,05 \%$ que fue establecido.

De acuerdo a la variable precio de la soja, banano y cacao se puede concluir lo siguiente según se muestra en la tabla 1.

- Con respecto a la soja, el coeficiente del precio es de 1,37. De acuerdo a la teoría económica, se podría concluir que el aumento promedio de $1 \%$ en el precio de la soja, conllevará a un aumento de la demanda del $137 \%$ de toneladas a nivel mundial. Es decir, este tipo de bien es considerado como un bien de Giffen.

- En el caso del banano el coeficiente del precio es de -3.72. De acuerdo a la teoría económica se podría concluir que el aumento promedio de $1 \%$ en el precio del banano, conllevará a una disminución de la demanda del $372 \%$ de toneladas a nivel mundial.

- Finalmente para el cacao, el coeficiente del precio es de -0.49. De acuerdo a la teoría económica se podría concluir que el aumento en promedio del $1 \%$ en el precio del cacao, conllevará a una disminución de la demanda del $49 \%$ de toneladas a nivel mundial.

Como se muestra en la tabla 2, se procedió a corregir las regresiones de los productos como arroz, maíz y trigo, de los betas que no aportaron a la función de demanda planteada con respecto al precio.

Se corrió una nueva regresión sin la variable del costo de vida y se concluyó que sin esta variable

TABLA 2. COEFICIENTES DEL PRECIO DE LOS PRODUCTOS SIN COSTO DE VIDA

\begin{tabular}{lrrrrrrr} 
& COEF. & STD. ERR. & $\boldsymbol{Z}$ & $\mathbf{P}>|\mathbf{Z}|$ & \multicolumn{2}{c}{ [95\% CONF . INTERVAL] } & R-CUADRADO \\
\hline log_precio_arroz & 1.654 .373 & 2.621 .698 & 0.63 & 0.528 & -348.406 & 6.792 .807 & $0 \%$ \\
\hline log_precio_maíz & 1.656 .346 & 3.118 .307 & 0.53 & 0.595 & -4.455 .424 & 7.768 .116 & $0 \%$ \\
\hline log_precio_trigo & 7.687 .128 & 4.156 .464 & 1.85 & 0.064 & -0.4593916 & 1.583 .365 & $0 \%$ \\
\hline log_precio_soja & 2.670 .435 & 2.583 .652 & 1.03 & 0.301 & -239.343 & 7.734 .301 & $58 \%$ \\
log_precio_banano & -3.621 .095 & 1.157 .163 & -3.13 & 0.002 & -5.889 .092 & -1.353 .097 & $21 \%$ \\
log_precio_cacao & 0.2868274 & 0.2582409 & 1.11 & 0.267 & -0.2193155 & 0.7929703 & $66 \%$ \\
\hline
\end{tabular}


TABLA 3. COEFICIENTES DEL COSTO DE VIDA DE LOS PRODUCTOS

\begin{tabular}{lrrrrrrr} 
& COEF. & STD. ERR. & $\mathbf{Z}$ & P>|z| & \multicolumn{2}{c}{ [95\% CONF .INTERVAL] } & R -CUADRADO \\
\hline log_costo de vida_arroz & 1.048 .703 & 0.7983082 & 1.31 & 0.189 & -0.5159524 & 2.613 .358 & $0 \%$ \\
\hline log_costo de vida_maíz & -1.397 .284 & 5.044 .274 & -0.28 & 0.782 & -1.128 .388 & 8.489 .311 & $0 \%$ \\
\hline log_costo de vida_trigo & -5.937 .752 & 6.890 .173 & -0.09 & 0.931 & -1409827 & 1.291 .071 & $0 \%$ \\
\hline log_costo de vida_soja & 0.1096544 & 0.0607068 & 1.81 & 0.071 & -0.0093288 & 0.2286376 & $94 \%$ \\
\hline log_costo de vida_banano & 0.4801683 & 0.2110782 & 2.27 & 0.023 & 0.0664626 & 0.893874 & $32 \%$ \\
\hline log_costo de vida_cacao & 0.1773902 & 0.0374679 & 4.73 & 0 & 0.1039545 & 0.2508259 & $79 \%$ \\
\hline
\end{tabular}

los resultados fueron los mismos. Es decir, en los coeficientes del arroz, maíz y trigo con respecto a la variable precio, los nuevos betas no aportan a la nueva regresión, dado que su $\mathrm{P}>|z|$ fue alto y un R cuadrado bajo.

Con respecto al costo de vida que se muestra en la tabla 3, se podría concluir que los productos que aportan al modelo son la soja, banano y cacao.

Planteándose lo siguiente, como resultado:

- El coeficiente del costo de vida de la soja es de 0.10 . Con respecto a la teoría económica, se podría concluir que el aumento promedio de un $1 \%$ en términos mundiales, conllevará a un aumento de un $10 \%$ en la demanda de la soja.

- El coeficiente del costo de vida del banano es de 0.48 . De acuerdo a la teoría económica, se podría concluir que el aumento promedio de $1 \%$ en términos mundiales, conllevará a un aumento del $48 \%$ en la demanda del banano.

- El coeficiente del costo de vida del cacao es de 0.17 . De acuerdo a la teoría económica, se podría concluir que el aumento promedio de $1 \%$ en términos mundiales, conllevará a un aumento de un $17 \%$ en la demanda del cacao.

Con respecto al PIB per-cápita que se muestra en la tabla 4, se podría concluir que:
Los productos que aportan al modelo son la soja, banano y cacao; mostrándose los siguientes resultados:

- El coeficiente del PIB per-cápita es de 0.92. De acuerdo a la teoría económica, se podría concluir que el aumento en promedio del 1\% del ingreso, conllevará a una disminución de la demanda de la soja en un $92 \%$. Razón por la cual es considerado un bien inferior en la economía a nivel mundial.

- El coeficiente del PIB per-cápita es de 0.13. De acuerdo a la teoría económica se podría concluir que el aumento en promedio del 1\% del ingreso, conllevará al aumento en el 13\% de las cantidades demandadas del banano. Motivo por el cual es considerado un bien normal en la economía a nivel mundial.

- El coeficiente del PIB per-cápita es de 0.54. De acuerdo a la teoría económica se puede concluir que el aumento en promedio del $1 \%$ del ingreso, conllevará a una disminución en un $54 \%$ de la demanda del cacao. Situación que lleva a considerarlo como un bien inferior en la economía a nivel mundial, según es mostrado en la tabla 5.

Con el fin de corregir las regresiones de los productos arroz, maíz y trigo, de los betas que

TABLA 4. COEFICIENTES DEL PIB PER-CÁPITA DE LOS PRODUCTOS

\begin{tabular}{lrrrrrrr} 
& COEF. & STD. ERR. & $\mathbf{Z}$ & P>|Z] & \multicolumn{2}{c}{ [95\% CONF .INTERVAL] } & R-CUADRADO \\
\hline log_pib_arroz & 3.660 .889 & 4.085 .642 & 0.9 & 0.37 & -4.346 .823 & 116.686 & $0 \%$ \\
\hline log_pib_maíz & -8.708 .139 & 2.712 .622 & -0.32 & 0.748 & -6.187 .455 & 4.445 .827 & $0 \%$ \\
\hline log_pib_trigo & -2.170 .578 & 2.390 .675 & -0.09 & 0.928 & -4.902 .694 & 4.468 .578 & $0 \%$ \\
\hline log_pib_soja & -0.9299413 & 0.1535768 & -6.06 & 0 & -1.230 .946 & -0.6289363 & $94 \%$ \\
\hline log_pib_banano & 0.134926 & 0.5958445 & 0.23 & 0.821 & -1.032 .908 & 130.276 & $32 \%$ \\
\hline log_pib_cacao & -0.5478078 & 0.1984687 & -2.76 & 0.006 & -0.9367993 & -0.1588164 & $79 \%$ \\
\hline
\end{tabular}


no aportaron la función de demanda planteada, con respecto al PIB per-cápita , se corrió una nueva regresión sin la variable costo de vida dado su baja significancia y R cuadrado bajo en el modelo general como se mostró en la tabla 5.

A partir de ello, se concluyó que sin esta variable los resultados fueron los mismos. Es decir los betas del arroz, maíz y trigo se siguieron comportando de igual manera con $\mathrm{R}$ cuadrado bajo como se mostró en la tabla 5 .

Finalmente, al comparar los resultados de las demandas de los productos primarios y secundarios se puede observar que la soja y el cacao son considerados bienes inferiores y el banano como un bien normal durante el período de tiempo analizado.

De acuerdo a los resultados, el cacao es considerado un bien inferior, ya que, ante un aumento del ingreso en términos mundiales, da como consecuencia una disminución de las cantidades demandas.

Sin embargo, de acuerdo a los resultados, el banano es considerado un bien normal, es decir que un aumento en los ingresos en términos mundiales, da como consecuencia un aumento de las cantidades demandadas.

\section{CONCLUSIONES}

Con respecto al segundo objetivo perseguido, se realizó el análisis de la demanda de los seis productos en función de las variables precio, costo de vida y PIB per cápita y se concluyó que solo los productos agrícolas soja, banano y cacao resultaron significativos en cada regresión y por lo tanto son los que aportan al modelo general.

En el tercer objetivo, se planteó comparar los resultados obtenidos diferenciando los productos primarios y secundarios. Se conoce que cacao y banano son considerados productos suntuarios y que se pudo contrastar únicamente con la soja que salió representativa en cuanto a los bienes primarios.
A partir de lo anterior, se puede decir que el banano y el cacao cumplen la función de demanda con respecto a que el aumento del precio disminuye las cantidades demandadas. Sin embargo, la soja no cumple la teoría de la demanda.

En cuanto a la elasticidad, los productos soja y cacao son considerados bienes inferiores y el banano es considerado un bien normal.

\section{RECOMENDACIONES}

Se recomienda que para determinar los precios se implementen otras metodologías además de la planteada en la investigación que sirvió de base a este artículo.

Finalmente, sería enriquecedor para corroborar lo investigado, ampliar el campo de estudio a la producción de otros países utilizando bienes suntuarios. Ello permitiría comparar el comportamiento en distintas culturas y economías.

\section{REFERENCIAS BIBLIOGRÁFICAS}

Albert, P. C., y Gómez, A. C. (1996). Productos típicos, territorio y competitividad. Agricultura y sociedad, 80, 57-82.

Banco Mundial. (2014). Informe sobre el desarrollo mundial 2014. Relaciones Internacionales. Washington D. C.: Grupo del Banco Mundial.

Camacho, F. (2014). Determinación delaselasticidadesingreso de la demanda de los principales productos agrícolas ecuatorianos de la balanza comercial respecto a productos agrícolas de primera necesidad mundial en tiempos de recesión. (Tesis de Maestría). Escuela Superior Politécnica del Litoral, Guayaquil, Ecuador.

Di Girolamo, G. (1992). El escenario agrícola mundial en los años noventa. Revista de la CEPAL, 08, 101-123.

Von Hesse, M. (1994). Políticas públicas y competitividad de las exportaciones agrícolas. Revista de la CEPAL, 53, 129-146. 